\title{
Connexin 32 and its derived homotypic gap junctional intercellular communication inhibit the migration and invasion of transfected HeLa cells via enhancement of intercellular adhesion
}

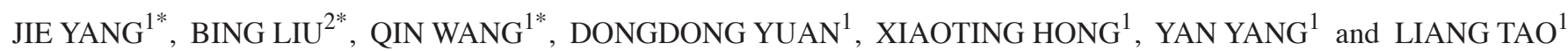 \\ ${ }^{1}$ Department of Pharmacology, Zhongshan School of Medicine, Sun Yat-Sen University, Guangzhou 510080; \\ ${ }^{2}$ Department of Pharmacology, School of Pharmacy, Guangdong Pharmaceutical University, Guangzhou 510006, P.R. China
}

Received March 21, 2011; Accepted June 7, 2011

DOI: $10.3892 / \mathrm{mmr} .2011 .509$

\begin{abstract}
The effects of connexin (Cx) and its derived homotypic gap junctional intercellular communication (GJIC) between tumor cells on the invasion of metastatic cancers and the underlying mechanisms remain unclear. In this study, we investigated the influence of Cx32 and the homotypic GJIC mediated by this $\mathrm{Cx}$ on the migration, invasion and intercellular adhesion of transfected HeLa cells. The expression of Cx32 significantly increased cell adhesion and inhibited migration and invasion. The inhibition of GJIC by oleamide, a widely used GJIC inhibitor, reduced the enhanced adhesion and partly reversed the decreased migration and invasion that had been induced by Cx32 expression. Blockage of the p38 and extracellular signal-regulated kinase 1 and 2 mitogenactivated protein kinase (ERK1/2 MAPKs) pathways using their specific inhibitors attenuated the effects of $\mathrm{Cx} 32$, but not those of GJIC, on cell adhesion, migration and invasion. These results indicate that the homotypic GJIC mediated by $\mathrm{Cx} 32$, as well as the $\mathrm{Cx}$ itself, inhibit cell migration and invasion, most likely through the elevation of intercellular adhesion. The suppressive effect of Cx32 on the migration and invasion of cancer cells, but not that of its derived homotypic GJIC, partly depends on the activation of the p38 and the ERK1/2 MAPKs pathways.
\end{abstract}

\section{Introduction}

The most life-threatening attribute of cancer cells is their ability to metastasize to distant organs, a process in which invasion and migration are required. It is well known that

Correspondence to: Dr Liang Tao, Department of Pharmacology, Zhongshan School of Medicine, Sun Yat-Sen University, Guangzhou 510080, P.R. China

E-mail: taol@mail.sysu.edu.cn

*Contributed equally

Key words: connexin32, homotypic gap junctional intercellular communication, migration, invasion, adhesion, mitogen-activated protein kinase, HeLa cells cancer cell migration is essential for invasion (1), which is a complex process regulated by matrix-degrading proteinases, integrins and cell-adhesion molecules that mediate tumor cell adhesion (2). As adhesion and migration are vital events in cancer progression and particularly in invasion, the regulation of cancer cell adhesion and migration represents an attractive therapeutic target against invasive cancer.

Gap junctions composed of connexins (Cxs) mediate the direct intercellular movement of cytoplasmic signaling molecules. There are approximately $21 \mathrm{Cx}$ isoforms, each of which forms channels with distinct regulatory and permeability characteristics (3). Intercellular signaling transmission mediated by $\mathrm{Cx}$ channels is important in human diseases, including cancer (4).

There is conflicting evidence regarding the effects of Cxs and their derived gap junctional intercellular communication (GJIC) on the invasion of metastatic cancers. Nicolson et al revealed a correlation between the loss of GJIC and spontaneous metastatic potential in rat $13762 \mathrm{NF}$ mammary adenocarcinoma cell sublines (5). In addition, Bodenstine et al recently reported the GJIC-dependent suppression of breast cancer cell metastasis (6). However, Fujimoto et al revealed that Cx32 suppressed the invasion of Caki-1 cells (a metastatic renal cell carcinoma cell line) partly via the inhibition of the Src-Stat3-VEGF signaling pathway, whereas treatment with 18- $\alpha$-glycyrrhetinic acid (a specific inhibitor against GJIC) had no impact on Src activity, indicating that GJIC was not involved in Cx32-regulated tumor cell invasion (7). Thus, the exact action of Cxs and GJIC on the invasive capacity of tumor cells and the underlying mechanisms remain unclear.

Several reports have indicated that Cxs act as adhesion molecules and mediate malignant tumor cell adhesion without requiring gap junction formation $(8,9)$. However, other studies have revealed that heterotypic GJIC between tumor cells and surrounding epithelial cells contributes to tumor cell adhesion and migration (10), and have confirmed the adhesive role of homotypic GJIC between tumor cells in many tumor cell lines (11). Nevertheless, it is unclear whether homotypic GJIC-dependent intercellular adhesion correlates with the regulation of tumor cell migration and invasion.

In this study, the effects of $\mathrm{Cx} 32$ and its derived homotypic GJIC on the invasive properties of HeLa cells [a highly 
invasive cervical cancer cell line (12)] were investigated. It was noted that both Cx32 and its derived homotypic GJIC suppressed cell migration and invasion, while enhancing intercellular adhesion. Inhibitors of the p38 and extracellular signal-regulated kinase 1 and 2 mitogen-activated protein kinase (ERK1/2 MAPK) pathways partly diminished the effects of $\mathrm{Cx} 32$ on cell adhesion, migration and invasion, but not those of GJIC, indicating that various mechanisms are responsible for the actions of this $\mathrm{Cx}$ and its derived GJIC. These results indicate that GJIC mediated by $\mathrm{Cx} 32$, as well as the $\mathrm{Cx}$ itself, inhibit cell migration and invasion, possibly by elevating intercellular adhesion. The suppressive effect of Cx32, but not that of its derived GJIC, on the migration and invasion of cancer cells partly depends on the activation of the p38 and ERK1/2 MAPKs pathways.

\section{Materials and methods}

Materials. Cell culture reagents were obtained from Invitrogen. G418, hygromycin and doxycycline were obtained from Calbiochem. Calcein-acetoxymethyl ester was purchased from Molecular Probes. Oleamide was from Sigma. Mouse anti-hemiglutinin (HA) clone HA-7 immunoglobin G ( $\mathrm{IgG}$, alkaline phosphatase-conjugated goat anti-mouse IgG and a-tubulin polyclonal antibodies were obtained from Sigma. The p38 MAPK inhibitor, SB203580, and the ERK1/2 MAPK inhibitor, PD98059, were obtained from Merck. Anti-ERK1/2 MAPK, anti-phospho-ERK1/2 MAPK (Thr202/Tyr204), anti-p38 MAPK and anti-phospho-p38 MAPK (Thr180/ Tyr182) antibodies, and horseradish peroxidase (HRP)-labeled anti-rabbit secondary antibody were purchased from Cell Signaling. All other reagents were purchased from Sigma unless stated otherwise.

Cell culture. HeLa cells that express Cx32 under the control of a bidirectional tetracycline-inducible promoter were described and characterized previously (13). In this stably transfected cell line, the Cx32 coding sequence is followed by a sequence coding for a thrombin-cleavable $\mathrm{COOH}$-terminal epitope tag consisting of an HA epitope followed by a 6 x (His-Asn) sequence. Connexin expression was induced with doxycycline $(1 \mu \mathrm{g} / \mathrm{ml})$ for $48 \mathrm{~h}$. Cells were cultured at $37^{\circ} \mathrm{C}$ and $5 \%$ carbon dioxide $\left(\mathrm{CO}_{2}\right)$ in Dulbecco's modified Eagle's medium (DMEM) supplemented with $10 \%$ fetal bovine serum, $100 \mu \mathrm{g} / \mathrm{ml} \mathrm{G} 418$ sulfate and $200 \mu \mathrm{g} / \mathrm{ml}$ hygromycin B.

Assays for cell growth/sulforhodamine B (SRB) assay. Changes in the growth of the HeLa cells were measured using a SRB colorimetric assay (14). Briefly, cells subjected to various treatments that match the conditions of invasion assays were grown in 96-well plates (see Cell invasion and migration assay). The culture medium was aspirated and the cells were fixed by the addition of $1 \mathrm{ml}$ of $10 \%$ cold trichloroacetic acid per well at $4{ }^{\circ} \mathrm{C}$ for $30 \mathrm{~min}$, then washed 5 times with deionized water and left to dry at room temperature overnight. Next, $1 \mathrm{ml}$ of $0.4 \%$ SRB in $1 \%$ acetic acid solution was added to each well and the plate was left to dry again at room temperature. SRB was removed and the plates were washed 5 times with $1 \%$ acetic acid. SRB bound to the cells was solubilized with $150 \mu \mathrm{l}$ Tris-buffer $(10 \mu \mathrm{M}, \mathrm{pH} 10.5)$ per well and the plates were left on a plate shaker for $10 \mathrm{~min}$. Absorbance was quantified using the Sunrise ${ }^{\mathrm{TM}}$ plate reader 5082 (Tecan Group Ltd., Grödig, Austria) at a wavelength of $570 \mathrm{~nm}$.

Cell invasion and migration assay. The effect of Cx32 expression on the invasion of transfected HeLa cells was determined using modified Boyden chambers with transwell (Corning Costar Corp., Cambridge, MA, USA) inserts with polycarbonate membrane filters with $8-\mu \mathrm{m}$ diameter pores (15). The filters were pre-coated with $50 \mu \mathrm{l}$ of Matrigel $(1.25 \mathrm{mg} /$ $\mathrm{ml})$. HeLa cells were serum-starved for $18 \mathrm{~h}$ in serum-free DMEM. The serum-starved cells were trypsinized and resuspended in DMEM, 5x $10^{4}$ cells in $100 \mu \mathrm{l}$ of serum-free DMEM were added to the upper chamber of each well, and $600 \mu \mathrm{l}$ of DMEM with $10 \%$ fetal bovine serum were placed in the bottom chamber. After $24 \mathrm{~h}$ at $37^{\circ} \mathrm{C}$, the non-invaded cells on the upper membrane surface were removed with a cotton swab. The invaded cells were fixed with $4 \%$ paraformaldehyde for $30 \mathrm{~min}$ and stained with $0.1 \%$ crystal violet for $30 \mathrm{~min}$. High-power fields $(n=8)$ of cells were counted in each well using an inverted microscope at x200 magnification. Invasion was expressed as the relative invasive rate, which is calculated as the invasive rate of the treated group (number of invaded cells/total cell number) divided by that of the untreated group. Meanwhile, an equal number of cells of the second group (treated and untreated) were seeded into 96-well plates at a density of $5 \times 10^{5}$ cells in $100 \mu \mathrm{l}$ of serum-free DMEM per well, to match the conditions of the cell-number invasion assays. The protocol of the migration assay was the same as that of invasion assay, except that no Matrigel was used.

Short-term aggregation assay. Confluent HeLa cells were incubated in $\mathrm{Ca}^{2+} / \mathrm{Mg}^{2+}$-free Hank's Buffered Salt Solution containing $1 \mathrm{mM}$ ethylenediaminetetraacetic acid (EDTA) for $15 \mathrm{~min}$. The cells were then dissociated mechanically with a Pasteur pipette to ensure maximum separation, centrifuged at $500 \mathrm{rpm}$ for $5 \mathrm{~min}$ and resuspended in fresh serum-free DMEM. Resuspension and centrifugation were repeated twice to remove EDTA. Runs containing cell clumps of $>1 \%$ of the population were discarded. A total of $4 \times 10^{5}$ cells were plated in 24-well plates cultured with $400 \mu \mathrm{l}$ of serum-free DMEM in each well. The plates were then placed on a rotary shaker (89 rpm) at $37^{\circ} \mathrm{C}$ in a $\mathrm{CO}_{2}$ incubator for $30 \mathrm{~min}$ and fixed by $40 \mu \mathrm{l}$ of $4 \%$ paraformaldehyde. Cellular clusters consisting of $>10$ cells were considered aggregates (8). The extent of aggregation was represented by the average percentage of aggregated cells in aggregates per field.

Dye-coupling detection by 'parachute' assay and flow cytometry. The so-called 'parachute' assay for GJIC was performed as described by Koreen et al (13). Donor and receiver cells were grown to confluence. The donor cells were doublelabeled with $5 \mu \mathrm{M}$ CellTracker ${ }^{\mathrm{TM}} \mathrm{CM}-\mathrm{DiI}$, a membrane dye that does not spread to gap junction-coupled cells, and with $5 \mu \mathrm{M}$ calcein-acetoxymethyl ester, which is converted intracellularly into calcein, a gap junction-permeable dye. The donor cells were then trypsinized and seeded onto the receiver cell monolayer at a 1:150 donor/receiver ratio. The donor cells were allowed to attach to the monolayer of receiver cells and 
form gap junctions for $4 \mathrm{~h}$ at $37^{\circ} \mathrm{C}$, and were then examined with an Olympus IX71 fluorescence microscope. For each experimental condition, we determined the average number of receiver cells containing dye per donor cell and normalized it to that of the control cultures.

To detect GJIC between suspended cells in the transwell chambers, we employed a dye-loading technique using flow cytometry as described previously (16) with a few modifications. Briefly, cells were loaded with $5 \mu \mathrm{M}$ of the green fluorescent dye calcein-acetoxymethyl ester, which is permeable through gap junctions, as mentioned above, and could be read on the flow cytometer. After incubation for $30 \mathrm{~min}$, stained cells were incubated with recipient unstained cells that were not treated with dye at a fixed ratio of 1:5 in the upper chambers of the transwells. The total cell number and density were equal to that of the invasion assay. After co-culture of these stained and unstained cells for $1 \mathrm{~h}$, the cells were washed with phosphate buffered saline (PBS) supplemented with $5 \%$ fetal bovine serum, then fixed in $4 \%$ cold paraformaldehyde and analyzed by flow cytometry. GJIC was represented by the calcein geometric mean fluorescence intensity (MFI) of recipient unstained populations. To confirm that calcein dye transfer was strictly dependent on GJIC and not due to the non-specific uptake of dye, pinocytosis or lysosomal transfer, stained and unstained cells were co-cultured in the presence of $50 \mu \mathrm{M}$ of oleamide, a GJIC blocker (17).

Western blotting. Western blotting protocols were as in previous studies (18). Briefly, cell lysates were separated by sodium dodecyl sulfate polyacrylamide gel electrophoresis in $10 \%$ Tris-glycine gels and transferred to a nitrocellulose (NC) membrane. Cx32 expression was evaluated on total cellular lysate by mouse anti-HA clone HA-7 IgG (U.S. Zymed) diluted $1: 800$ in $2.5 \%(\mathrm{w} / \mathrm{v})$ skimmed dry milk in Tris-buffered saline Tween-20 (TBST; 0.01 mol/1 PBS, pH 7.4, and $0.05 \%(\mathrm{v} / \mathrm{v})$ Tween-20) and incubated overnight at $4^{\circ} \mathrm{C}$. The blot was then incubated with alkaline phosphataseconjugated goat anti-mouse $\operatorname{IgG}$ as the secondary antibody at a 1:1,600 dilution for $1 \mathrm{~h}$ at room temperature. Equal loading of proteins was confirmed by probing with $\beta$-tubulin diluted $1: 10,000$ in TBST/5\% milk for $1 \mathrm{~h}$ at room temperature, and then probed with secondary antibody under the same conditions. For Western blot analysis of p38, phospho-p38, ERK1/2 and phospho-ERK1/2, all proteins were transferred to a NC membrane and blocked with $5 \%$ bovine serum albumin (BSA) in TBST. Blots were probed with specific antibodies raised to $\mathrm{p} 38$, phospho-p38, ERK1/2 and phospho-ERK1/2 (diluted with 5\% BSA to $1: 1,000$ for p38 and phospho-p38 and 1:1,000 for ERK1/2 and phospho-ERK1/2). Non-phosphorylated MAPK bands were chosen as loading controls for the activation of MAPKs. Membranes were probed with HRP-labeled anti-rabbit secondary antibody (diluted with 5\% BSA to 1:2,000; all antibodies were from Cell Signaling). Antibody binding was visualized using an enhanced chemiluminescence detection kit (ECL; Amersham International PLC, UK). All Western blotting exposures were in the linear range of detection, and the intensities of the resulting bands were quantified by Quantity One software on a GS-800 densitometer (Bio-Rad).

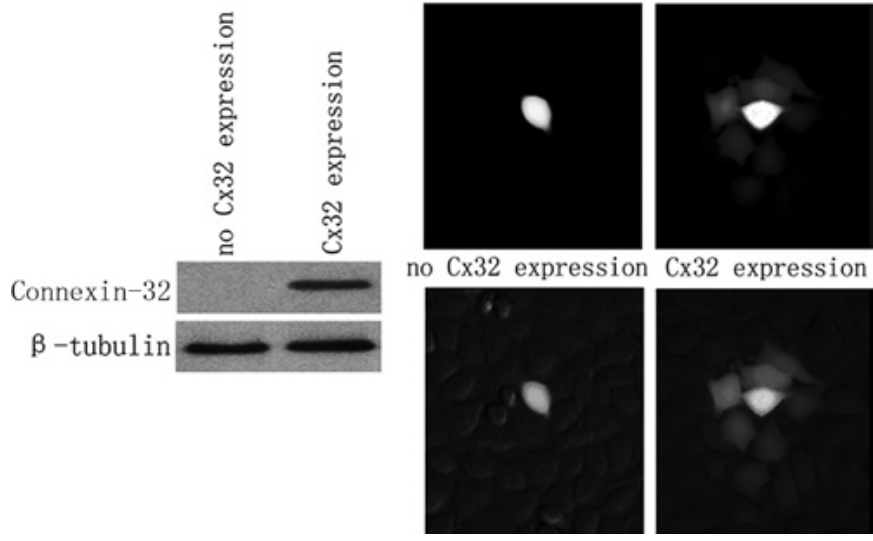

Figure 1. Doxycycline induction of connexin expression. Doxycycline $(1 \mu \mathrm{g} /$ $\mathrm{ml}$ ) induced $\mathrm{Cx} 32$ expression, as shown by Western blotting (left panel). Dye coupling through $\mathrm{Cx} 32$-derived gap junctions, as shown by parachute dye coupling assay (right panel). Magnification, x200.

Statistical analysis. Data were statistically analyzed using the unpaired Student's t-test at a significance level P-value of $<0.05$ and are presented as the means \pm standard error (SE), using Sigma Plot software (Jandel Scientific).

\section{Results}

Effect of Cx32 expression on HeLa cell invasion. To establish whether $\mathrm{Cx} 32$ expression regulates tumor cell promotion, we studied the effect of forced Cx32 expression on the migratory and invasive potential of cancer cells using HeLa cells transfected with Cx32 cDNA. The overexpression of $\mathrm{Cx} 32$ induced by doxycycline $(1 \mu \mathrm{g} / \mathrm{ml})$ and GJIC revealed by the parachute assay are shown in Fig. 1.

Fig. 2A illustrates the effect of doxycycline on the invasion of HeLa cells without transfection with the Cx32-containing plasmid (wild-type HeLa cells). As shown in Fig. 2A, doxycycline itself $(1 \mu \mathrm{g} / \mathrm{ml})$ had no unexpected effects on the invasion of wild-type HeLa cells. Fig. 2B shows that Cx32 expression induced by doxycycline diminished cell invasion and the invasive rate of HeLa cells by $\sim 55.7 \%$ compared to that of doxycycline-untreated cells (non-expression of Cx32). As indicated in Fig. 2C, Cx32 expression significantly suppressed HeLa cell migration through membranes that were not coated with Matrigel.

To exclude the possibility that the effect of Cx32 expression on cell migration and invasion occurred through an unspecific cytotoxicity-related mechanism, the growth rate of HeLa cells was determined using an SRB colorimetric assay. As shown in Fig. 2D, there were no statistically significant differences between the cell growth rates of cells expressing Cx32 and those cells not expressing Cx32, indicating that Cx32 expression had no cytotoxic effect on the cells.

Effects of both Cx32 and its derived homotypic GJIC on adhesion, migration and invasion of HeLa cells. To determine whether Cx32 and/or its derived homotypic GJIC are involved in the regulation of the invasive capacity of tumor cells, the adhesion, migration and invasion of HeLa cells was assayed in the presence or absence of oleamide, a widely accepted GJIC inhibitor. Oleamide was not cytotoxic and had no effect on the 
A
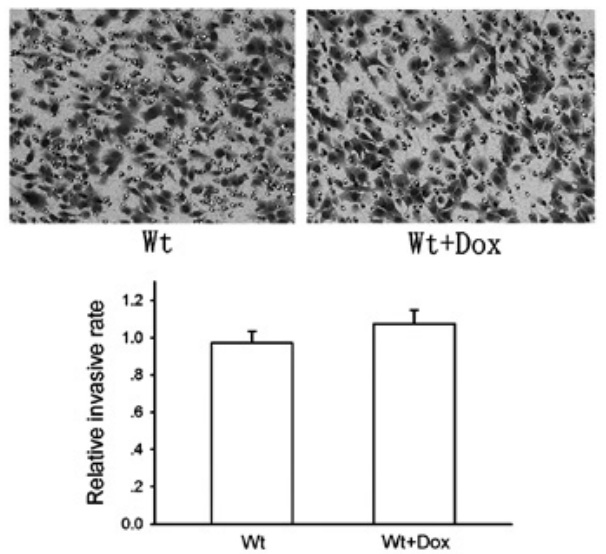

C

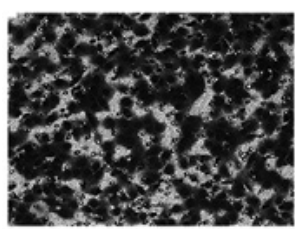

no Cx32 expression $\mathrm{Cx} 32$ expression

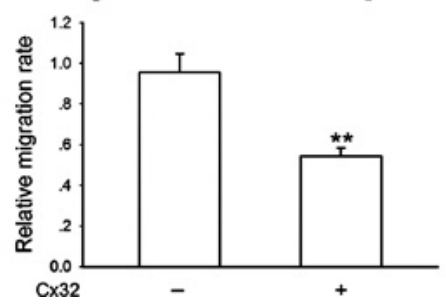

B
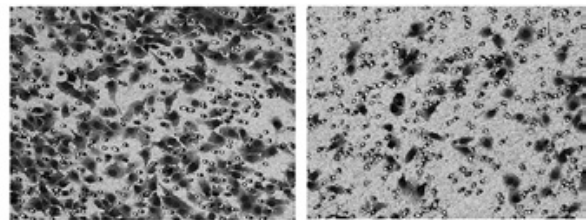

no $\mathrm{Cx} 32$ expression $\mathrm{Cx} 32$ expression

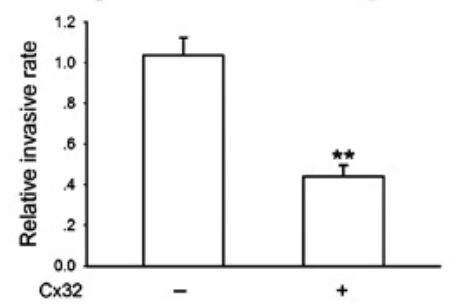

D

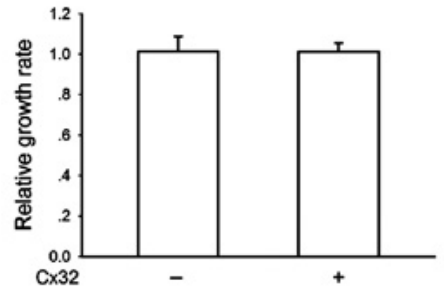

$* * \mathrm{p}<0.01$

Figure 2. Cx32 expression inhibited the invasive potential of HeLa cells. (A) Doxycycline (Dox) had no impact on the invasion of wild-type HeLa cells (Wt) which were not transfected with the plasmid coding for Cx32. (B) Cx32 expression significantly inhibited the invasive potential of transfected HeLa cells. (C) Cx32 expression substantially inhibited the migration of transfected HeLa cells. (D) Cx32 expression had no influence on the proliferation of transfected HeLa cells. Bars represent the means \pm SE from four to six independent experiments.

A

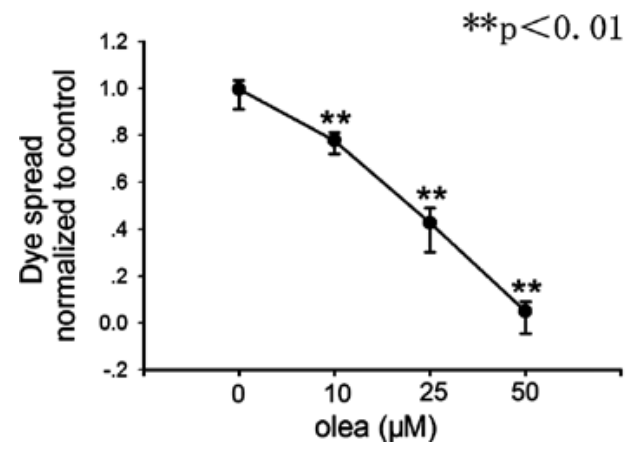

B

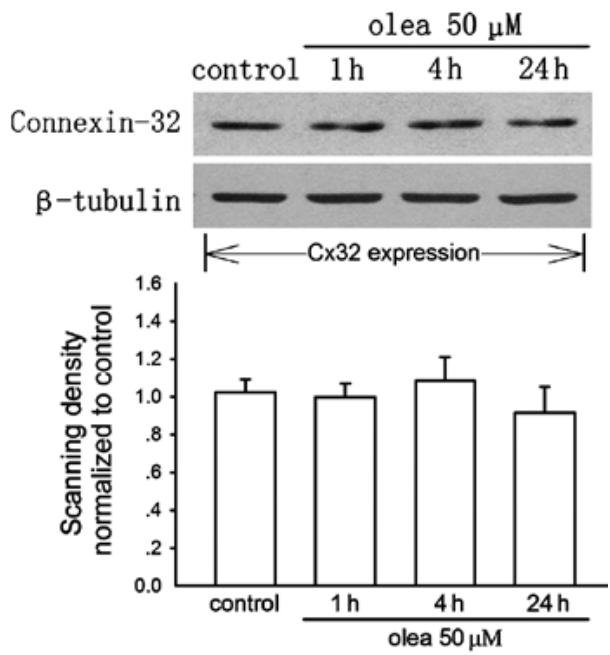

Figure 3. Oleamide (olea) diminished homotypic GJIC, but had no effect on Cx32 protein levels during its persistent treatment for up to $24 \mathrm{~h}$. (A) GJIC was assessed as the average number of receiver cells containing calcein from each donor cell, normalized to the Cx32 expression group without oleamide treatment. Dye spread of cells treated with a range of oleamide concentrations. Data points represent the means \pm SE from five independent experiments. (B) Western blotting showed no effect of oleamide on Cx32 protein level. Bar graphs are derived from densitometric scanning of the blots. Bars represent the means \pm SE from four independent experiments.

invasive capacity of HeLa cells at the concentrations used in the present study (data not shown). The results of the parachute assay indicate that oleamide inhibited GJIC in a concentration- dependent manner (Fig. 3A). Oleamide $(50 \mu \mathrm{M})$ diminished GJIC by $95 \%$ (Fig. 3A), but it did not change Cx32 protein levels during persistent treatment for up to $24 \mathrm{~h}$ (Fig. 3B). 


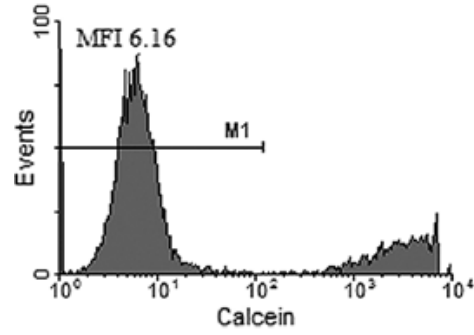

no Cx32 expression

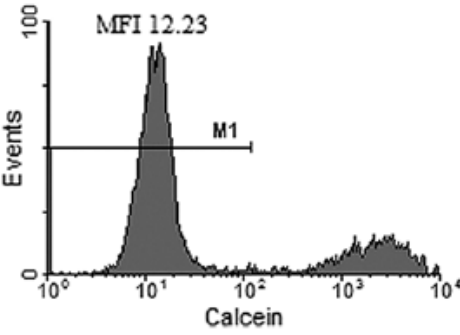

Cx32 expression

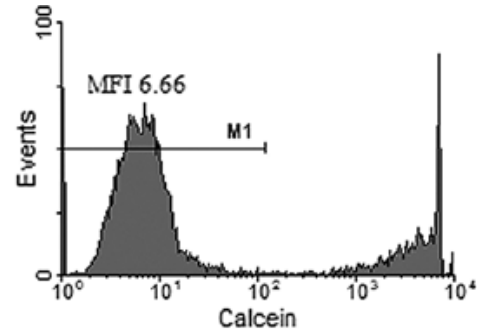

Cx32 expression+olea
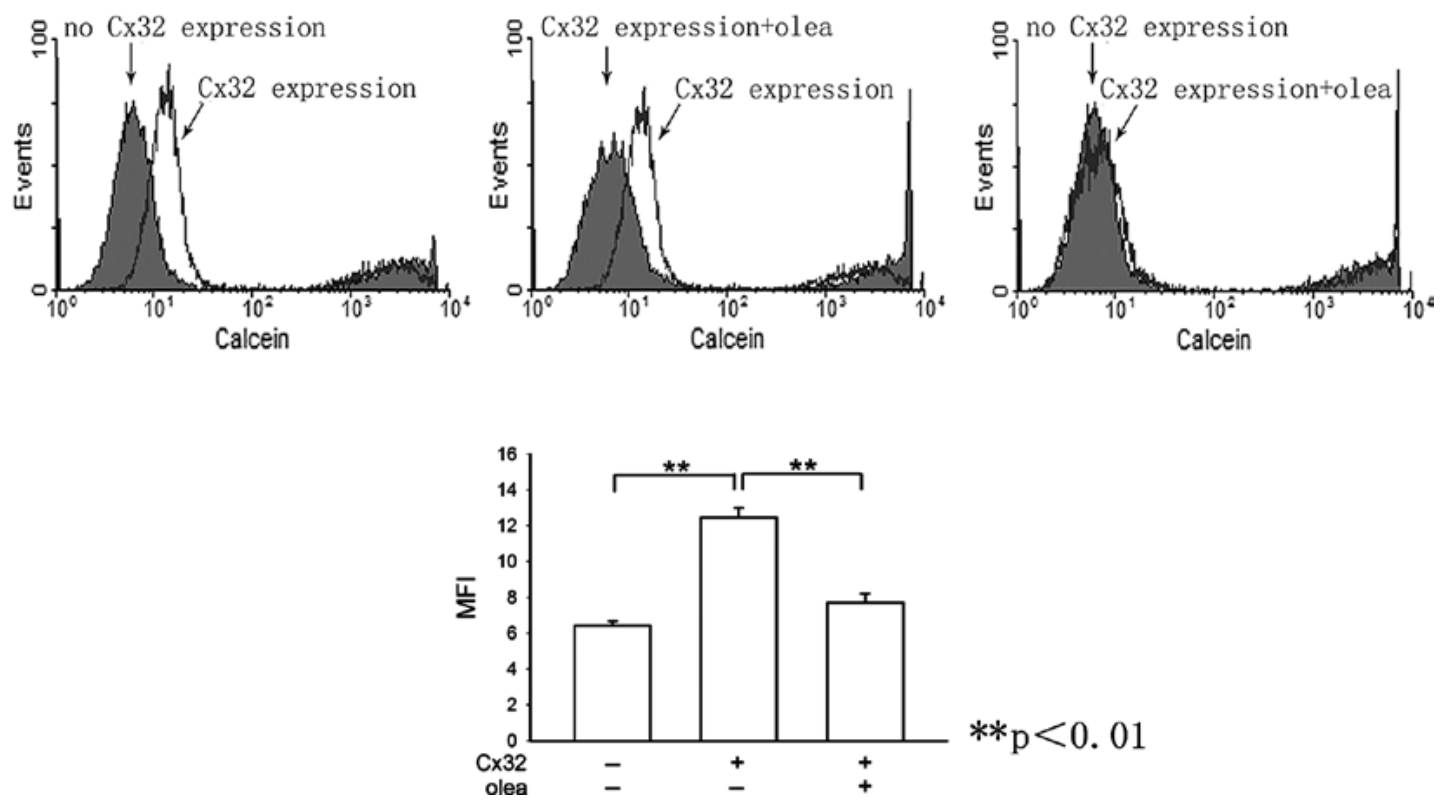

Figure 4. Flow cytometrical determination of the coupling of suspended cells in the upper chambers of Transwell. Cells were loaded with green fluorescent calcein and co-incubated with other unstained recipient cells at a fixed ratio of 1:5. After $1 \mathrm{~h}$ of co-incubation, the calcein mean fluorescence intensity (MFI) of recipient unstained populations was analyzed by flow cytometry. The increase in the MFI of recipient unstained populations reflected dye transfer through homotypic GJIC from stained cells. Oleamide, a specific GJIC blocker, was used to ascertain whether the intercellular dye transfer was specifically dependent on homotypic GJIC. The lower histograms are overlays to demonstrate the shift in MFI of recipient unstained cells on co-culture with calcein-labeled cells. The bottom bars represent the means \pm SE from four independent experiments.

Flow cytometry was used to determine GJIC between suspended transfected HeLa cells in the upper chamber of transwell inserts (see Materials and methods). The high sensitivity of the dye transfer flow cytometric assay allows for a precise evaluation of the amount of transferred dye in recipient unstained cells through the analysis of their MFI (19). As shown in Fig. 4, the MFI of recipient unstained populations of HeLa cells expressing Cx32 (middle panel) was substantially increased compared to that of cells without Cx32 expression (left panel), indicating the presence of homotypic GJIC in Cx32-expressing cells during cell invasion. Treatment of the cells expressing Cx32 with $50 \mu \mathrm{M}$ of oleamide (right panel) significantly reversed the MFI increase of recipient unstained populations, which confirms that the dye transfer was dependent on GJIC and indicates that oleamide specifically inhibited homotypic GJIC.

Fig. 5 shows that oleamide $(50 \mu \mathrm{M})$ partly reversed the effect of Cx32 expression on cell adhesion (Fig. 5A), migration (Fig. 5B) and invasion (Fig. 5C). The adhesive capacity of HeLa cells not expressing Cx32 was significantly lower than that of cells expressing $\mathrm{C} \times 32$ after oleamide $(50 \mu \mathrm{M})$ treatment. On the contrary, the migratory and invasive capacities of HeLa cells not expressing Cx32 were significantly greater than those of cells expressing Cx32 after oleamide treatment. These results suggest that the increased intercellular adhesion and the decreased cell migration and invasion induced by Cx32 expression occur partly through a GJIC-dependent mechanism; a GJIC-independent mechanism is also indicated. Thus, both Cx32 and its derived homotypic GJIC are critically involved in the regulation of the adhesion, migration and invasion of HeLa cells.

p38 and ERK1/2 MAPKs pathways are involved in the effects of CX32, but not in those of its derived homotypic GJIC, on the adhesion, migration and invasion of transfected HeLa cells. To investigate the molecular mechanism by which Cx32 expression influences the adhesive, migratory and invasive phenotypes of HeLa cells, we examined whether Cx32 expression regulated the activities of the p38 and ERK1/2 MAPKs pathways, which are closely correlated with the adhesive and migratory potential of cancer cells (20).

The effect of Cx32 expression on the activities of p38 and ERK1/2 MAPKs, which were represented by the levels of their phosphorylated forms, is illustrated in Fig. 6. In 


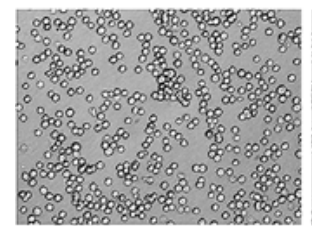

no $\mathrm{Cx} 32$ expression

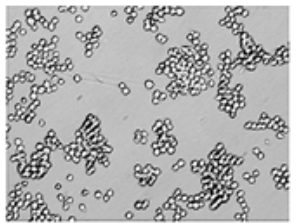

Cx32 expression

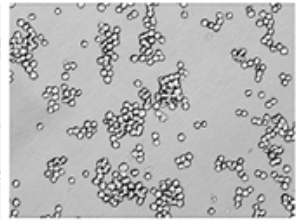

Cx32 expression+olea

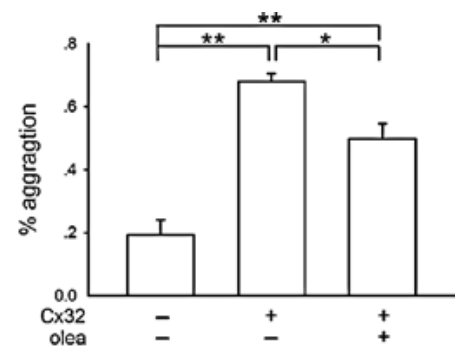

B

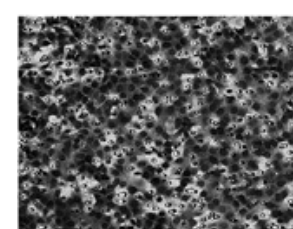

no Cx32 expression

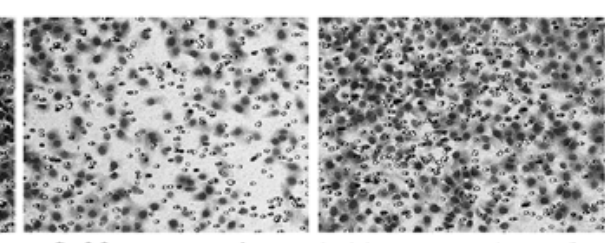

Cx32 expression Cx32 expression+olea

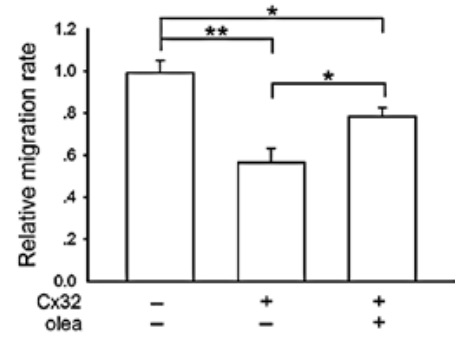

C
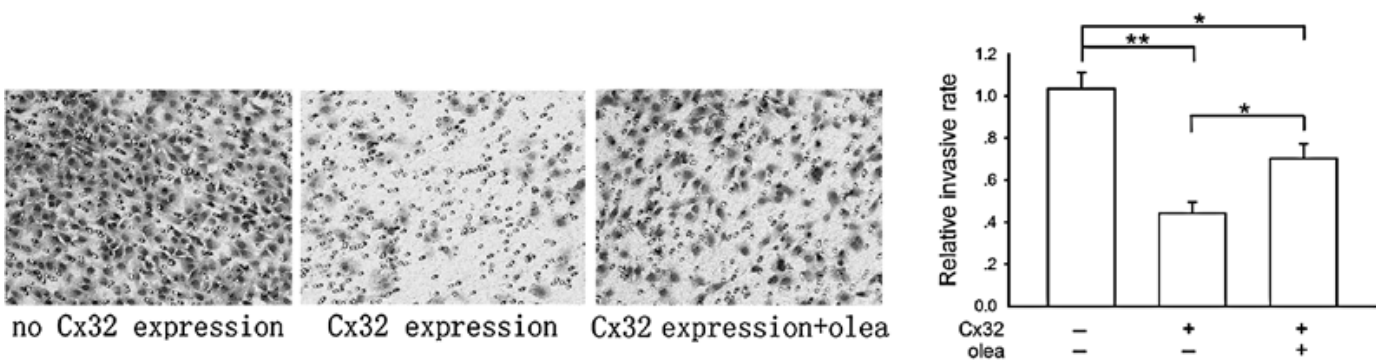

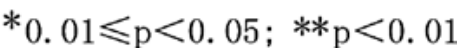

Figure 5. Cx32 and its derived homotypic GJIC on (A) intercellular adhesion, (B) migration and (C) invasion of transfected HeLa cells. Bars represent the means \pm SE from five to six independent experiments.

wild-type HeLa cells, doxycycline $(1 \mu \mathrm{g} / \mathrm{ml})$ did not alter the levels of phosphor-p38 (p-p38) and phosphor-ERK1/2 (p-ERK1/2), indicating that it had no effects on the activities of p38 and ERK1/2 MAPKs (Fig. 6A). As shown in Fig. 6B, Cx32 expression significantly enhanced the activities of p38 and ERK1/2 MAPKs, as evidenced by a substantial increase in the levels of p-p38 and p-ERK1/2. The inhibition of GJIC by oleamide $(50 \mu \mathrm{M})$ did not affect the activation of the $\mathrm{p} 38$ and ERK1/2 MAPKs by Cx32 expression. Pre-treatment of wild-type HeLa cells with $50 \mu \mathrm{M}$ of oleamide for $1 \mathrm{~h}$ had no effect on p38 and ERK1/2 MAPKs activation (Fig. 6C). These results suggest that $\mathrm{Cx} 32$ expression enhances the activities of the p38 and ERK1/2 MAPKs pathways through a GJICindependent mechanism.

Furthermore, we examined whether SB203580 (25 $\mu \mathrm{M})$ and PD98059 $(25 \mu \mathrm{M})$, the specific inhibitors of the p38 and ERK1/2 MAPKs, respectively, could reverse the effects of Cx32 on cell adhesion, migration and invasion.

Pre-treatment of the cells with SB203580 or PD98059 for $1 \mathrm{~h}$ significantly reduced the activities of the p38 and ERK1/2 MAPKs (Fig. 7A). Moreover, treatment of the HeLa cells expressing Cx32 with SB203580 or PD98059 reversed the effects of Cx32 on adhesion (Fig. 7B), migration (Fig. 7C) and invasion (Fig. 7D) to an extent that was indistinguishable from HeLa cells not expressing Cx32.
Consistent with the report of Ramer et al (21), treatment of HeLa cells not expressing Cx32 with SB203580 or PD98059 did not affect adhesion and migration (data not shown). Both SB203580 and PD98059 had no impact on the growth of wild-type HeLa cells as assessed by SRB (data not shown), suggesting that the effects of these inhibitors on adhesion and migration are unrelated to cell growth.

The results indicate that $\mathrm{Cx} 32$, but not its derived homotypic GJIC, increases the adhesion and inhibits the migration and invasion of HeLa cells, mainly through the activation of the p38 and ERK1/2 MAPKs pathways.

\section{Discussion}

This study demonstrates the anti-invasive effect of Cx32 expression in transfected HeLa cells. Our results indicate that the overexpression of $\mathrm{Cx} 32$ increases intercellular adhesion and suppresses the migration and invasion of HeLa cells. These effects are partly diminished by the application of oleamide, which fully suppresses GJIC, but does not alter Cx32 protein levels. As opposed to previous studies, which reveal a Cx32induced suppressive effect on the invasion of tumor cells in a manner independent of GJIC (7), the present study provides the first evidence for the involvement of $\mathrm{Cx} 32$ and its derived homotypic GJIC in the supression of HeLa cell invasion. 
A

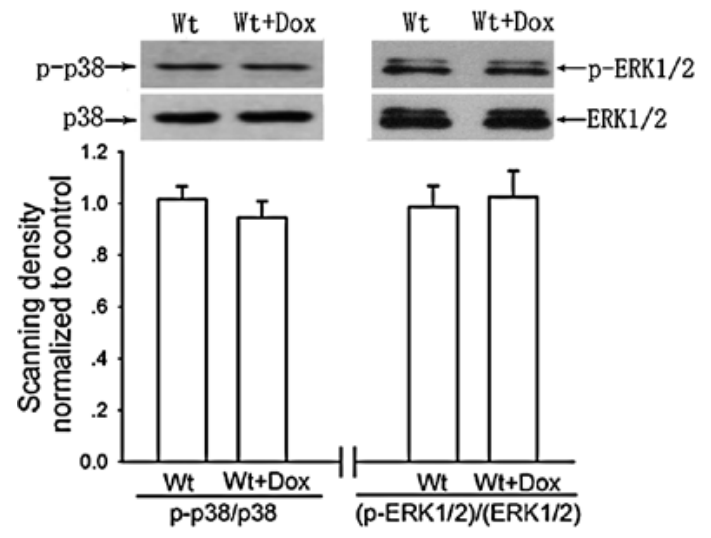

C

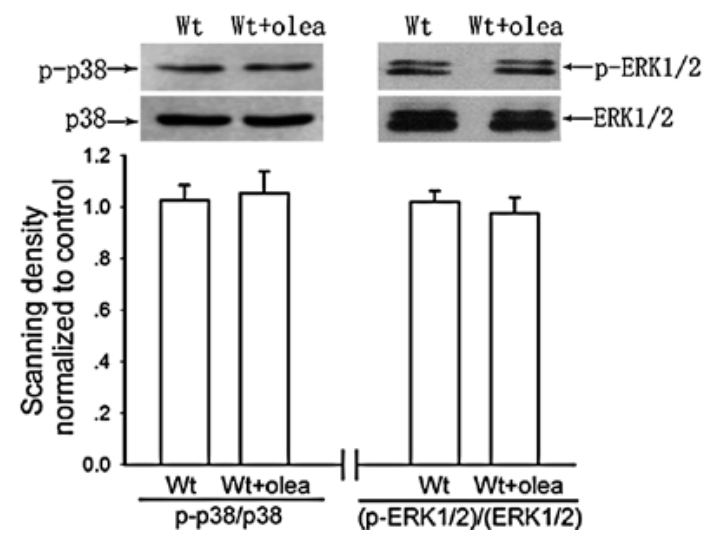

B
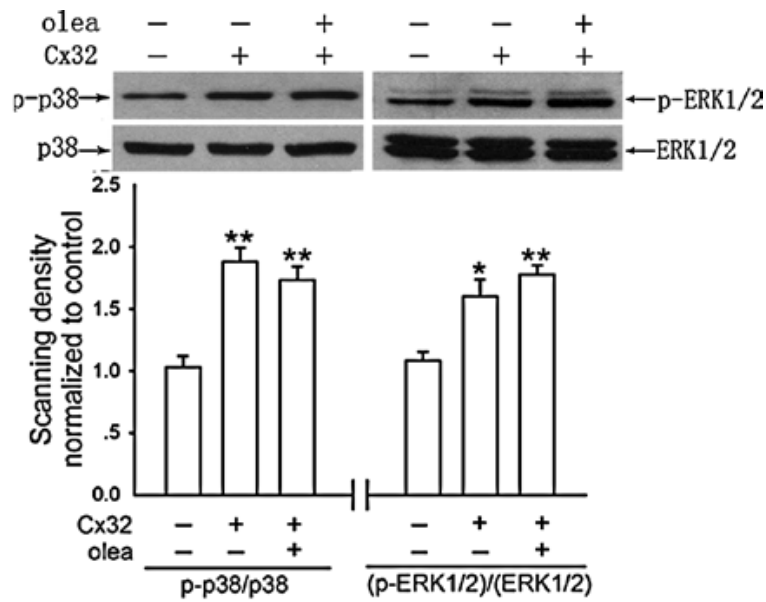

$* 0.01 \leqslant \mathrm{p}<0.05 ; * * \mathrm{p}<0.01$

Figure 6. Cx32 independently of GJIC prominently activates the p38 and ERK1/2 MAPKs. (A) Doxycycline (Dox) had no effect on the activities of the p38 and ERK1/2 MAPKs in wild-type HeLa cells (Wt) determined by Western blotting. Bar graphs are derived from densitometric scanning of the blots. Bars are the means \pm SE from four independent experiments. (B) Cx32, but not homotypic GJIC, activates the p38 and ERK1/2 MAPKs. The cells were pre-treated with $50 \mu \mathrm{M}$ of oleamide (a specific inhibitor against GJIC, olea) for $1 \mathrm{~h}$. To analyze the phosphorylation of the p38 and ERK1/2 MAPKs, immunoblots were probed with antibodies directed against the phosphorylated forms of the p38 and ERK1/2 MAPKs. Equal loading of lysates was ensured by probing membranes with antibodies directed against the total p38 or ERK1/2 MAPKs. Bar graphs are derived from densitometric scanning of the blots. Bars represent the means \pm SE from four independent experiments. (C) Oleamide has no impact on the activities of the p38 and ERK1/2 MAPKs. Wild-type HeLa cells were pre-treated with oleamide for $1 \mathrm{~h}$ and the phosphorylated forms of the $\mathrm{p} 38$ and ERK1/2 MAPKs were determined by Western blotting. Bar graphs are derived from densitometric scanning of the blots. Bars represent the means \pm SE from three independent experiments.

Numerous studies have revealed the different roles of Cx32 expression in the regulation of invasion in various types of tumor cells. For example, in human metastatic renal carcinoma cells, $\mathrm{Cx} 32$ has been demonstrated to act as a tumor suppressor gene against invasion and metastasis, independently of the formation of GJIC (7). On the contrary, the expression of $\mathrm{Cx} 32$, which is mainly retained in the cytoplasm, has been shown to promote the progression of human ductal breast cancer cells (22) and hepatocellular carcinoma cells (23). In addition, it has been reported that forced Cx32 expression does not affect the invasive potency of glioma cells, while it significantly increases the dye coupling between these cells (8). These results, in combination with the data presented in this study, suggest the cell type specificity for the suppressive effects of $\mathrm{Cx} 32$ expression on the invasive potency of cancer cells.

Prior reports have indicated that Cxs regulate the invasion of tumor cells through various mechanisms. For example, the forced expression of $\mathrm{Cx} 26$ in HepG2 cells reduces their invasiveness through the suppression of matrix metallopeptidase-9 activity (24), and Cx32 expression in human renal carcinoma cells has been shown to inhibit cell invasion by blocking the
Src-Stat3-VEGF signaling pathway (7). The present study shows for the first time that the suppressive effect of $\mathrm{C} \times 32$ on the migration and invasion of HeLa cells partly depends on the activation of $\mathrm{p} 38$ and ERK1/2 MAPK pathways.

Numerous studies have indicated the vital contribution of the MAPK pathway, as well as the p38 and ERK1/2 MAPK pathways, in the regulation of migration and invasion of various cancer cell lines. However, the effects of these signaling pathways on the migration and invasion differ depending on the cell type tested. For example, constitutive p38 MAPK activity has been confirmed to be crucial for the maintenance of breast cancer invasive phenotype via the stabilization of uPA and uPAR expression (25). Other studies have also revealed the positive regulatory role of $\mathrm{p} 38$ or ERK1/2 MAPK pathways in the invasive phenotype of human hepatocellular carcinoma cells and oral squamous cell carcinoma cells, respectively $(26,27)$. However, cisplatin and cannabinoids have been shown to exert their anti-invasive properties on human cervical carcinoma cells (HeLa and C33A) and human lung carcinoma cells (A549) via the activation of the p38 and ERK1/2 MAPK pathways, respectively $(21,12)$. In our study, the activities of $\mathrm{p} 38$ and ERK1/2 MAPKs were also 
A
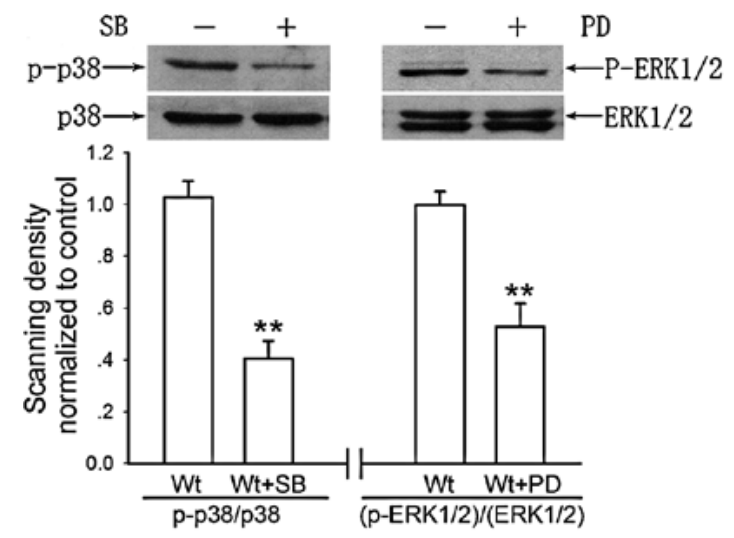

C

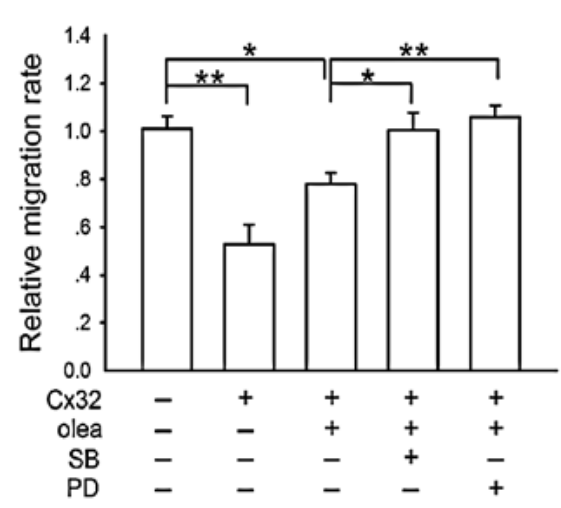

B

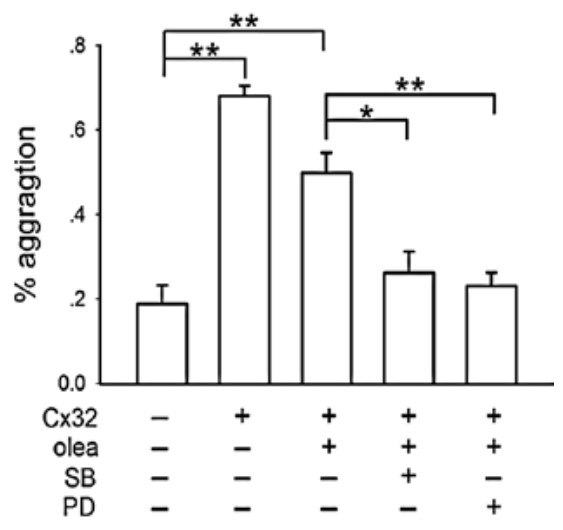

D

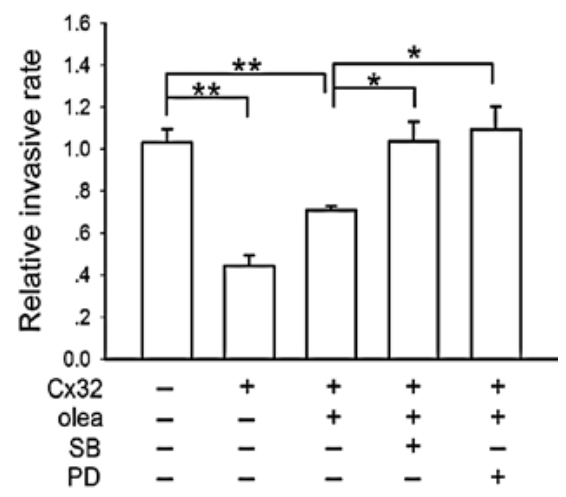

$* 0.01 \leqslant \mathrm{p}<0.05 ; * * \mathrm{p}<0.01$

Figure 7. Involvement of the 38 and ERK1/2 MAPKs pathways in the effects of Cx32, but not in those of homotypic GJIC, on the adhesion, migration and invasion of HeLa cells. (A) Inhibitory effect of the p38 and ERK1/2 MAPKs inhibitors, SB203580 (SB) and PD98059 (PD), respectively. Whole-cell lysates prepared from wild-type HeLa cells with SB203580 or PD98059 treatment for $1 \mathrm{~h}$ were analyzed for expression of phosphorylated and total MAPKs by Western blotting. Bars represent the means \pm SE from four independent experiments. Cx32, but not homotypic GJIC, increased (B) intercellular adhesion and decreased (C) migration and (D) invasion through the activation of $\mathrm{p} 38$ and ERK1/2 MAPKs pathways. Cells were pre-treated with oleamide to inhibit GJIC and then treated with $25 \mu \mathrm{M}$ of SB203580 or $25 \mu \mathrm{M}$ of PD98059 for aggregation, migration and invasion assays. Bars represent the means \pm SE from five to seven independent experiments.

shown to be negatively correlated with the invasive potency of HeLa cells. This evidence also indicates cell type specificity for the suppressive effects of MAPK pathway activation on the invasive potency of cancer cells. Further studies are required to demonstrate the cell type specificities for the effect of Cx32 on invasion as well as on $\mathrm{p} 38$ and ERK1/2 MAPK pathways.

The role of the p38 and ERK1/2 MAPK pathways in the regulation of cell adhesion has been demonstrated by several studies $(28,29)$. Most recently, the ERK MAPK pathway was shown to play a novel role in the dynamic remodeling of focal adhesions and the actin cytoskeleton, which are crucial determinants of cell motility (30). However, no studies have addressed the correlations between the p38 and ERK1/2 MAPK pathways and CX-mediated changes in adhesion, migration and invasion. It was observed that the Cx32-mediated adhesive, anti-migratory and anti-invasive effects were reversed by the inactivation of the p38 and ERK1/2 MAPKs pathways. This indicates that the concomitant activation of the p38 and ERK1/2 MAPKs pathways is critical to the enhancement of adhesion and the suppression of migration and invasion induced by $\mathrm{Cx} 32$ expression. Nevertheless, Cx-dependent adhesion was likely modulated by other adhesion molecules (8), especially E-cadherin. The loss of E-cadherin-mediated adhesion led to the increased motility of tumor cells (31). Since E-cadherin expression can be induced by Cxs $(32,33)$, our study cannot fully exclude the possibility that E-cadherin may be involved in the Cx32related increase in cell adhesion.

This study indicates that Cx32 and its derived homotypic GJIC enhance cell adhesion and reduce migration and invasion. Pre-treatment of the cells expressing Cx32 with oleamide, a GJIC inhibitor, decreases adhesion and increases migration and invasion without affecting the activities of the p38 and ERK1/2 MAPKs, suggesting that the GJIC-related effects do not occur through these pathways.

Although Cxs establish adhesive interactions independent of GJIC $(8,9)$, certain reports have indicated that GJIC is sufficient for tumor cell adhesion $(11,34)$. El-Sabban and Pauli reported a critical interdependence of heterotypic adhesion and GJIC between endothelial cells and lung metastatic cancer cells (35). This type of heterotypic GJIC may trigger adhesion between tumor cells and host endothelial cells, thus contributing to the detachment of malignant cells from the tumor mass. However, consistent with the report that the Cx43-mediated homotypic GJIC between glioblastoma cells supported intercellular adhesion (11), the present study 
indicates that homotypic GJIC is critical for the intercellular adhesion of tumor cells. Most importantly, we observed that homotypic GJIC-mediated adhesion, probably because it restricts the detachment of tumor cells from each other, is related to the decreased migration and invasion of tumor cells. Since homotypic GJIC does not alter the activities of the p38 and ERK1/2 MAPKs pathways, the mechanism by which it affects adhesion, migration and invasion requires further investigation.

\section{Acknowledgements}

This study was supported by the National Natural Science Foundation of China (No. 30973434 and No. 30901807), and a grant for the development of important new drugs from the Ministry of Health of China (No. 2009ZX09303-007).

\section{References}

1. Aznavoorian S, Murphy AN, Stetler-Stevenson WG and Liotta LA: Molecular aspects of tumor cell invasion and metastasis. Cancer 71: 1368-1383, 1993.

2. Chapman HA: Plasminogen activators, integrins, and the coordinated regulation of cell adhesion and migration. Curr Opin Cell Biol 9: 714-724, 1997.

3. Harris AL: Connexin channel permeability to cytoplasmic molecules. Prog Biophys Mol Biol 94: 120-143, 2007.

4. Dobrowolski R and Willecke K: Connexin-caused genetic diseases and corresponding mouse models. Antioxid Redox Signal 11: 283-295, 2009.

5. Nicolson GL, Dulski KM and Trosko JE: Loss of intercellular junctional communication correlates with metastatic potential in mammary adenocarcinoma cells. Proc Natl Acad Sci USA 85: 473-476, 1988.

6. Bodenstine TM, Vaidya KS, Ismail A, et al: Homotypic gap junctional communication associated with metastasis suppression increases with PKA activity and is unaffected by PI3K inhibition. Cancer Res 70: 10002-10011, 2010.

7. Fujimoto E, Sato H, Shirai S, et al: Connexin32 as a tumor suppressor gene in a metastatic renal cell carcinoma cell line. Oncogene 24: 3684-3690, 2005.

8. Lin JH, Takano T, Cotrina ML, et al: Connexin 43 enhances the adhesivity and mediates the invasion of malignant glioma cells. J Neurosci 22: 4302-4311, 2002.

9. Cotrina ML, Lin JH and Nedergaard M: Adhesive properties of connexin hemichannels. Glia 56: 1791-1798, 2008.

10. Li Z, Zhou Z and Donahue HJ: Alterations in $\mathrm{Cx} 43$ and OB-cadherin affect breast cancer cell metastatic potential. Clin Exp Metastasis 25: 265-272, 2008

11. Oliveira R, Christov C, Guillamo JS, et al: Contribution of gap junctional communication between tumor cells and astroglia to the invasion of the brain parenchyma by human glioblastomas. BMC Cell Biol 6: 7, 2005.

12. Ramer R and Hinz B: Inhibition of cancer cell invasion by cannabinoids via increased expression of tissue inhibitor of matrix metalloproteinases-1. J Natl Cancer Inst 100: 59-69, 2008.

13. Koreen IV, Elsayed WA, Liu YJ and Harris AL: Tetracyclineregulated expression enables purification and functional analysis of recombinant connexin channels from mammalian cells. Biochem J 383: 111-119, 2004.

14. Papazisis KT, Geromichalos GD, Dimitriadis KA and Kortsaris AH: Optimization of the sulforhodamine B colorimetric assay. J Immunol Methods 208: 151-158, 1997.

15. Zhu W, Ou Y, Li Y, et al: A small-molecule triptolide suppresses angiogenesis and invasion of human anaplastic thyroid carcinoma cells via down-regulation of the nuclear factorkappa B pathway. Mol Pharmacol 75: 812-819, 2009.

16. Bazarbachi A, Abou Merhi R, Gessain A, et al: Human T-cel lymphotropic virus type I-infected cells extravasate through the endothelial barrier by a local angiogenesis-like mechanism. Cancer Res 64: 2039-2046, 2004.
17. Guan X, Cravatt BF, Ehring GR, Hall JE, Boger DL, Lerner RA and Gilula NB: The sleep-inducing lipid oleamide deconvolutes gap junction communication and calcium wave transmission in glial cells. J Cell Biol 139: 1785-1792, 1997.

18. He B, Tong X, Wang L, et al: Tramadol and flurbiprofen depress the cytotoxicity of cisplatin via their effects on gap junctions. Clin Cancer Res 15: 5803-5810, 2009.

19. Fonseca PC, Nihei OK, Savino W, Spray DC and Alves LA: Flow cytometry analysis of gap junction-mediated cell-cell communication: advantages and pitfalls. Cytometry Part A 69A: 487-493, 2006.

20. Tremblay PL, Auger FA and Huot J: Regulation of transendothelial migration of colon cancer cells by E-selectin-mediated activation of p38 and ERK MAP kinases. Oncogene 25: 6563-6573, 2006.

21. Ramer R, Eichele K and Hinz B: Upregulation of tissue inhibitor of matrix metalloproteinases-1 confers the anti-invasive action of cisplatin on human cancer cells. Oncogene 26: 5822-5827, 2007.

22. Kanczuga-Koda L, Sulkowska M, Koda M, Rutkowski R and Sulkowski S: Increased expression of gap junction protein connexin 32 in lymph node metastases of human ductal breast cancer. Folia Histochem Cytobiol 45 (Suppl 1): 175-180, 2007.

23. Li Q, Omori Y, Nishikawa Y, Yoshioka T, Yamamoto Y and Enomoto K: Cytoplasmic accumulation of connexin32 protein enhances motility and metastatic ability of human hepatoma cells in vitro and in vivo. Int J Cancer 121: 536-546, 2007.

24. Yano T and Yamasaki H: Regulation of cellular invasion and matrix metalloproteinase activity in HepG2 cell by connexin 26 transfection. Mol Carcinog 31: 101-109, 2001.

25. Huang S, New L, Pan Z, Han J and Nemerow GR: Urokinase plasminogen activator/urokinase-specific surface receptor expression and matrix invasion by breast cancer cells requires constitutive p38alpha mitogen-activated protein kinase activity. J Biol Chem 275: 12266-12272, 2000.

26. Hsieh YH, Wu TT, Huang CY, Hsieh YS, Hwang JM and Liu JY: P38 mitogen-activated protein kinase pathway is involved in protein kinase alpha-regulated invasion in human hepatocellular carcinoma cells. Cancer Res 67: 4320-4327, 2007.

27. Chen PN, Hsieh YS, Chiang CL, Chiou HL, Yang SF and Chu SC: Silibinin inhibits invasion of oral cancer cells by suppressing the MAPK pathway. J Dent Res 85: 220-225, 2006.

28. Paine E, Palmantier R, Akiyama SK, Olden K and Roberts JD: Arachidonic acid activates mitogen-activated protein (MAP) kinase-activated protein kinase 2 and mediates adhesion of a human breast carcinoma cell line to collagen type IV through a p38 MAP kinase-dependent pathway. J Biol Chem 275: 11284-11290, 2000.

29. Fincham VJ, James M, Frame MC and Winder SJ: Active ERK/ MAP kinase is targeted to newly forming cell-matrix adhesions by integrin engagement and v-Src. EMBO J 19: 2911-2923, 2000.

30. Pullikuth AK and Catling AD: Extracellular signal-regulated kinase promotes Rho-dependent focal adhesion formation by suppressing p190A RhoGAP. Mol Cell Biol 30: 3233-3248, 2010.

31. Alt-Holland A, Shamis Y, Riley KN, DesRochers TM, Fusenig NE, Herman IM and Garlick JA: E-cadherin suppression directs cytoskeletal rearrangement and intraepithelial tumor cell migration in 3D human skin equivalents. J Invest Dermatol 128: 2498-2507, 2008.

32. Xu HT, Li QC, Zhang YX, Zhao Y, Liu Y, Yang ZQ and Wang EH: Connexin 43 recruits E-cadherin expression and inhibits the malignant behaviour of lung cancer cells. Folia Histochem Cytobiol 46: 315-321, 2008.

33. Yano T, Hernandez-Blazquez FJ, Omori Y and Yamasaki H: Reduction of malignant phenotype of HEPG2 cell is associated with the expression of connexin 26 but not connexin 32 . Carcinogenesis 22: 1593-1600, 2001.

34. Elias LA, Wang DD and Kriegstein AR: Gap junction adhesion is necessary for radial migration in the neocortex. Nature 448: 901-907, 2007.

35. El-Sabban ME and Pauli BU: Adhesion-mediated gap junctional communication between lung-metastatatic cancer cells and endothelium. Invasion Metastasis 14: 164-176, 1994. 